\title{
HEXAGONAL SYSTEMS OF SEVEN LINES IN A PLANE*
}

\author{
BY LOUISE D. CUMMINGS
}

1. Introduction. This paper concerns the determination of the non-equivalent systems of seven real lines in a plane when no three of the lines are co-punctual, and the investigation is limited to that subdivision of the problem where, in a system of seven lines, some six form a convex hexagon. It is shown that exactly eight non-equivalent arrangements exist, and incidentally the integers showing the numbers of polygons of $3,4,5,6,7$ sides which occur in each of the eight systems are tabulated. The method, developed by Professor H. S. White, of the unique characterization of a line by means of the contiguous line-segments in a system, has been used to determine a "mark" for every line in the eight systems. The seven marks of a system are employed to prove the non-equivalence of systems and to determine the substitution connecting two equivalent systems.

2. Basic Hexagon. Six lines in a plane, no three in any point, form 30 segments, and for the hexagonal subdivision here considered, divide the plane into one hexagon, six triangles, and nine quadrilaterals. For easier visualization, an irregular hexagon with sides produced indefinitely and with all 15 intersections in the finite plane is considered. The 30 segments of the six lines may be assigned to three classes, namely, 6 primary, separating the hexagon from the triangles, 12 secondary, separating the triangles from quadrilaterals, 12 tertiary, separating, each, two quadrilaterals. The nine quadrilaterals are separable into two types: (1) six quadrilaterals with sides two adjacent secondary segments and two adjacent tertiary segments, (2) three quadrilaterals with all sides tertiary segments. The 30 segments separate into five continuous broken lines as follows: the 6 primary segments bound the hexagon, the 12 secondary segments surround the six triangles, and the 12 tertiary segments separate into the three boundaries of the three quadrilaterals of the second type.

3. Notation and Method. When, in a set of seven lines, any six form a hexagon, that hexagon is utilized as unique initial figure.

* Presented to the Society, October 31, 1931. 
The six vertices in order on the hexagon are marked 1, 2, 3, 4, 5, 6 . On the broken line of the secondary segments insert the letters $a, b, c, d, e, f$, in order, $a$ being the vertex of the triangle whose base is the primary segment 12. Mark the three intersections of the three pairs of opposite sides of the hexagon $L, M, N$, in the same cyclic order, $L$ being the intersection of the pair of opposite sides 12 and 45. Mark the six intersections of the seventh line or secant as $p, q, r, s, t, u$. Name the six lines forming the hexagon $l_{1}, l_{2}, l_{3}, l_{4}, l_{5}, l_{6}$ and the seventh line $l_{7}$. Two subdivisions of the problem are considered: Case I and Case II.

CASE I. The seventh line crosses the hexagon, intersecting two primary segments, two secondary segments, and two tertiary segments. In the hexagon the two primary segments may be (i) adjacent sides, (ii) alternate sides, (iii) opposite sides.

CASE II. The seventh line lies outside the hexagon and intersects (i) six tertiary segments, (ii) four tertiary and two secondary segments, (iii) two tertiary and four secondary segments.

Case I (i). The seventh line enters the hexagon across a triangle, for example the triangle $a 12$, and cutting the segments $a 1,12$, emerges from the hexagon across the consecutive side 23 and must cut the segments $3 b, c L, f M$. In the pentagonal subdivision of seven real lines in a plane, $\mathrm{H}$. S. White has employed a unique mark for any secant in a system of lines by means of the contiguous line-segments which that secant crosses. Two segments are contiguous if they have a common extremity, and in determining the mark of a secant contiguous segments are written so as to form a continuous broken line. For example the line $l_{7}$ crosses the contiguous segments $a 1,12,23,3 b$ and two separate segments $c L, f M$, and the mark for the line $l_{7}$ is written $l_{7}(a 123 b \cdot c L \cdot f M)$ or for brevity $l_{7}(4 \cdot 1 \cdot 1)$. The line $l_{7}$ intersecting two consecutive sides of the basic hexagon divides the hexagon into a triangle and a heptagon. In this heptagon the seventh line and the initial six lines now occupy similar roles, each being a side of the heptagon, hence we know, without further examination, that all seven lines have the same mark $(4 \cdot 1 \cdot 1)$. This system designated as system (1) is identified by the characteristic $(4 \cdot 1 \cdot 1)^{7}$. 
CAse I (ii).* The seventh line or secant enters the hexagon across the segments $a 1,12$ but emerges across the side of the hexagon 34 , alternate to the side 12 , and entering the triangle $c 34$ may leave the triangle across $3 c$ or $4 c$. The path across $3 c$ is unique and the secant cuts the six segments $a 1,12,43,3 c, c L$, $f M$ and the mark of this line $l_{7}(a 12 \cdot 43 c L \cdot f M)$ is $l_{7}(3 \cdot 2 \cdot 1)$. This investigation is concerned primarily with the development of a necessary and sufficient test for the equivalence or non-

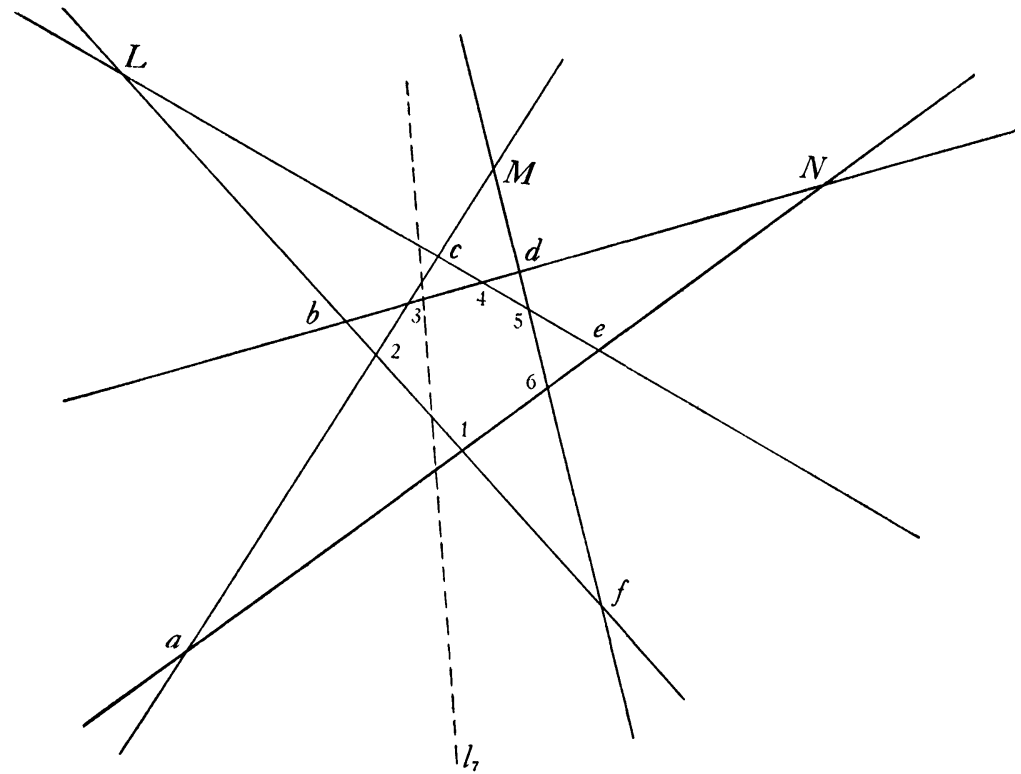

FIG. 1. System (2)

equivalence of two systems of seven real lines in a plane, where two systems are equivalent if a one-to-one relation exists between the lines and polygons of the two systems. The line $l_{7}(3 \cdot 2 \cdot 1)$ of system (2) cannot be transformed into any line with the mark (4.1.1) and therefore system (2) is not equivalent to system (1). However, for purposes of comparison with other systems, it is necessary in some cases to have all seven marks of each system and the marks of system (2) consist of four of this new kind $(3 \cdot 2 \cdot 1)$ and three of the first kind $(4 \cdot 1 \cdot 1)$, and system (2) has the characteristic $(4 \cdot 1 \cdot 1)^{3}+(3 \cdot 2 \cdot 1)^{4}$. If the line

* Figure 1. 
$l_{7}$ crosses the segments $a 1,12,34,4 c$ into the quadrilateral $4 c M d$, two paths of exit are possible, either across the side $c M$ adjacent to $4 c$ or across the segment $d M$ opposite to $4 c$, and the two paths must be examined. On the first path the six segments cut by the secant are $a 1,12,34,4 c, c M, M f$ and the mark of the secant is $l_{7}(a 12 \cdot 34 c M f)=(4,2)$. This mark does not occur in system (1) nor in system (2) and belongs to a new system (3) with the characteristic $(5 \cdot 1)+(4 \cdot 2)^{2}+(4 \cdot 1 \cdot 1)+(3 \cdot 2 \cdot 1)^{3}$. Examination of the second possible path across $4 c$ gives a system $\left(3^{\prime}\right)$ with the same characteristic as that derived for system (3). A comparison now of the seven marks of these two systems determines very easily the substitution $S$ which transforms system $\left(3^{\prime}\right)$ in to system (3), namely, $S \equiv(14)(23)(56)(a c)(b)(d f)(e)$ $(L N)(M)(p q)(r u)(s t)$.

CASE I (iii). The seventh line crosses that hexagon on two opposite sides 12 and 45, and the investigation of all possible paths shows the existence of three new non-equivalent systems (4), (5) and (6) tabulated below in Table I.

CASE II (i). The secant crosses six tertiary segments giving rise to two non-equivalent systems designated in the following table as (7) and (8). In system (7) the three Pascalian points $L, M, N$ all lie on the same side of the seventh line, and the seventh line crosses the three quadrilaterals of the second type and three consecutive quadrilaterals of the first type. In system (8) the points $L, M, N$ do not all lie on the same side of the seventh line, and the seventh line crosses the three quadrilaterals of the second type and three alternate quadrilaterals of the first type.

CASE II (ii). The seventh line crosses two secondary segments and then may cross four tertiary segments over two different paths, giving rise to two systems $\left(3_{1}\right)$ and $\left(3_{2}\right)$ each of which is equivalent to system (3). This result was to be expected since in system (3) a second hexagon, including the line $l_{7}$, exists, which may be used as basic hexagon. If the segments are renamed with respect to this second hexagon the secant line, now $l_{2}$, crosses four tertiary and two secondary segments.

CASE II (iii). The path of the seventh line across four secondary segments and two tertiary segments is unique and gives rise to one system $\left(2_{1}\right)$ which is equivalent to system (2), a result in 
agreement with the fact that system (2) contains also a second hexagon.

4. Table of Non-Equivalent Hexagonal Systems. In the following Table I, the marks for each of the eight non-equivalent hexagonal systems are given and also, incidentally, the actual polygonal division of the projective plane, satisfying of course the Euler equation

$$
s_{7}+s_{6}+s_{5}+s_{4}+s_{3}=\frac{n^{2}-n+2}{2}
$$

where $s_{i}$, the number of polygons of $i$ sides, is tabulated for each of the eight systems. The three non-equivalent systems, derived by $\mathrm{H}$. S. White in the pentagonal subdivision of his paper,* have been adjoined in Table I for convenience in comparison, and are designated here as (9), (10), (11).

\begin{tabular}{|c|c|c|c|c|c|c|c|c|c|c|c|c|}
\hline \multirow[t]{2}{*}{ SYSTEM } & \multicolumn{7}{|c|}{ CHARACTERISTICS } & \multicolumn{5}{|c|}{ POLYGONS } \\
\hline & $(6 \cdot 0)$ & $(5 \cdot 1)$ & $(4 \cdot 2)$ & $(4 \cdot 1 \cdot 1)$ & $(3 \cdot 3)$ & $(3 \cdot 2 \cdot 1)$ & $(2 \cdot 2 \cdot 2)$ & $s_{7}$ & so & $s_{6}$ & $s_{4}$ & $s_{3}$ \\
\hline (1) & & & & 7 & & & & 1 & & & 14 & 7 \\
\hline (2) & & & & 3 & & 4 & & & 1 & 1 & 13 & 7 \\
\hline (3) & & 1 & 2 & 1 & & 3 & & & 1 & 2 & 11 & 8 \\
\hline (4) & & & & 2 & & 4 & 1 & & & 3 & 12 & 7 \\
\hline (5) & 1 & 2 & & & 2 & 2 & & & & 5 & 8 & 9 \\
\hline (6) & & 2 & & & 1 & 4 & & & & 4 & 10 & 8 \\
\hline (7) & 5 & & 2 & & & & & & 1 & 5 & 5 & 11 \\
\hline \multirow[t]{2}{*}{ (8) } & & & 6 & & & & 1 & & 1 & 3 & 9 & 9 \\
\hline & & Pen & tagonal & Systems & & & & & & & & \\
\hline (9) & & & & & & 6 & 1 & & & 3 & 12 & 7 \\
\hline (10) & & & 2 & & 1 & 2 & 2 & & & 4 & 10 & 8 \\
\hline (11) & 3 & & & & 3 & & 1 & & & 6 & 6 & 10 \\
\hline
\end{tabular}

5. Properties shown by Table I. The numbers listed for the polygonal divisions of the plane for these eight hexagonal systems agree with the result of this investigation, that the eight systems are non-equivalent, and the same fact is true for the table of the pentagonal systems. However, a comparison of the two parts of the table shows that systems (6) and (10) have the same polygonal numbers $4,10,8$, and hence that equality of polygonal numbers is a necessary but not a sufficient condition for the equivalence of two systems. The characteristics of these two systems, however, show immediately that the two systems are non-equivalent. The non-equivalent systems (4) and (10) also have equality of polygonal numbers.

\footnotetext{
* The plane figure of seven real lines, this Bulletin, vol. 38 (1932), pp. 59-65.
} 
6. Conclusion. This method of analysis of straight-line nets by the contiguous segments, as herein extended to all the lines in the system, regardless of their relation to a pentagon, hexagon, or other basal polygon, is applicable to any number $n$ of straight lines and is even not restricted to the case that only $m=2$ lines shall pass through a point. The method furnishes a necessary and sufficient test for the equivalence or the non-equivalence of two systems of straight lines, and in the case of two equivalent systems this method simplifies the discovery of the substitution which transforms the one system into the other system.

Vassar College

\section{A PRACTICAL METHOD FOR THE MODULAR REPRESENTATION OF FINITE OPERA- TIONS AND RELATIONS*}

\section{BY B. A. BERNSTEIN AND NEMO DEBELY}

1. Introduction. In previous papers one of the writers developed a general theory for the concrete representation of arbitrary operations and relations in a finite class of elements. $\dagger$ Let $p$ be a prime, and let $a \bmod p$ denote the least positive integer obtained from integer $a$ by dropping multiples of $p$. Consider the function $f(x)$ given by

$$
f(x)=c_{0}+c_{1} x+\cdots+c_{p-1} x^{p-1}
$$

$\bmod p$,

where $x$ ranges over the complete system of $p$-residues $0,1, \cdots$, $p-1$, and where the coefficients $c_{i}$ are among the $p$-residues. The general theory is based on the fact that any unary operation in a class $K$ of $p$ elements, the operation satisfying the condition of closure, can be represented by a polynomial of form (1). But when the number of elements in $K$ is large, the calculation of (1) by the method of the general theory is very laborious, for the work involves, for a class of $p$ elements, the computation modulo $p$ of $p$ determinants each of order $p-1$. For an $m$-ary operation or an $m$-adic relation where $m>2$, the calculation of the representation by the method of the general theory is very laborious

* Presented to the Society, April 5, 1930.

$\uparrow$ See the Proceedings of the International Mathematical Congress, Toronto, 1924, p. 207, and this Bulletin, vol. 32, p. 533. 